\title{
Reading Brendan Behan, John McCourt (dir.)
}

\section{Chiara Rustici}

\section{(2) OpenEdition}

\section{Journals}

\section{Édition électronique}

URL : https://journals.openedition.org/etudesirlandaises/10413

DOI : 10.4000/etudesirlandaises. 10413

ISSN : 2259-8863

\section{Éditeur}

Presses universitaires de Caen

\section{Édition imprimée}

Date de publication : 31 décembre 2020

Pagination : 208-210

ISBN : 978-2-84133-996-9

ISSN : 0183-973X

\section{Référence électronique}

Chiara Rustici, «Reading Brendan Behan, John McCourt (dir.) », Études irlandaises [En ligne], 45-2 | 2020, mis en ligne le 31 décembre 2020, consulté le 22 novembre 2022. URL : http://

journals.openedition.org/etudesirlandaises/10413; DOI : https://doi.org/10.4000/etudesirlandaises. 10413

\section{(c) (i) (2)(2)}

Creative Commons - Attribution - Pas d'Utilisation Commerciale - Partage dans les Mêmes Conditions 4.0 International - CC BY-NC-SA 4.0

https://creativecommons.org/licenses/by-nc-sa/4.0/ 
(which the Catholic Church did not object to). This gave the male members of the working classes of the city an unprecedented and unequalled opportunity to play sport, develop masculine and parish identity and eventually articulate and mediate strong inner-city rivalries. As it was also used as a recruitment pool by senior rugby clubs of the city, Limerick developed a very specific rugby culture characterised by competitiveness and toughness which was unique in Ireland but could also be observed in Northern England and France.

Regarding its so-called “foreign” nature, O'Callaghan provides a wealth of evidence showing that the transformation of folk football into various specific sports codes was a gradual process, and that in the late $19^{\text {th }}$ century there were frequent overlaps and great fluidity between "native" and "foreign" games. For some of its devotees of the period, rugby was as valid a medium for expressing nationalistic sentiment as the GAA. The totemic GAA ban on "foreign" games was originally meant as a means of boosting interest in the Gaelic sport's association and attained extreme ideological importance only in the decades after independence: "attachment of politico-cultural significance to different sports in Ireland was an evolutionary, contested process, the success of which was both regionally and chronologically varied" (p. 143).

Thirdly, as a product of the Victorian public-school system, rugby theoretically served the function of educating the ideal imperial man and providing a model for moral gentlemanliness. However, O'Callaghan goes at great lengths to show that rugby in Limerick was and is much more competitive than the original amateur ideal would have allowed and that the manliness it was devised to engender took on a very different shape in Munster, where both players and spectators gave short shrift to the self-discipline the game is supposed to nurture. There again: "Manhood ideologies are not immutable constructs but are adaptations to social environments which are part of the material conditions of life in the various societies during particular time periods" (cited p. 118).

Therefore, likewise, the $21^{\text {st }}$ century characterisation of Munster rugby as a unique phenomenon built upon notions of community solidarity and humble social roots is only partially true if not entirely false. It was crafted by early $21^{\text {st }}$ century marketers to create some sense of belonging and uniqueness and ensure its success.

Still plenty more work to do by (sports) historians.

Laurent DANIEL

\section{Reading Brendan Behan, John McCourt (dir.), Cork, Cork University Press, 2019, $\mathrm{x}+250 \mathrm{p}$.}

L'ouvrage de John McCourt, Reading Brendan Behan, ne peut qu'être salué avec enthousiasme car il constitue une nouvelle, très attendue, contribution sur l'œuvre de l'un des auteurs irlandais les plus éclectiques, mais aussi l'un des moins étudiés. 
En effet, exception faite pour la monographie publiée en 2002 par John Brannigan, Brendan Behan: Cultural Nationalism and the Revisionist Writer (Dublin, Four Courts Press), Behan n'a pas fait l'objet d'études approfondies depuis près de vingt ans.

Certes, la critique doit faire face à quelques difficultés. On pense notamment à l'absence d'un corpus complet et définitif. La question de la collaboration ou de la coécriture d'un certain nombre de textes ainsi que l'absence d'une anthologie complète compliquent la tâche. De plus, la réputation de Behan et ses problèmes avec l'alcool ont souvent fait passer son rôle d'artiste au second plan. Pourtant l'œuvre de Behan mériterait plus d'attention.

McCourt part de ce postulat et cherche à rendre à Behan sa place d'auteur moderniste et expérimental. Reading Brendan Behan est bien organisé et les contributeurs sont éminents. On regrettera seulement que les contributions se concentrent, pour la plupart, sur les œuvres majeures de Behan, d'autant plus que McCourt rappelle en introduction la mise en circulation de matériel inédit en 2017 (p. 1).

En effet, les cinq premiers essais (sur les onze qui constituent le recueil) concernent principalement Borstal Boy. Bien que ces articles analysent le texte sous différents angles, on retrouve une volonté commune d'inscrire l'œuvre de Behan dans le courant moderniste, notamment en comparaison avec d'autres auteurs contemporains.

Brannigan, par exemple, refuse de lire l'œuvre de Behan exclusivement en relation au contexte politique irlandais et souligne les connexions et les affinités avec Joyce, Beckett, Genet ou encore Camus et Sartre. C'est notamment lors de ses séjours à Paris que Brendan Behan a pu retrouver la liberté intellectuelle nécessaire pour développer « les formes de son art» (p. 58) et s'interroger, entre autres, sur le rapport entre art et politique (p. 57).

Deirdre McMahon cherche également à souligner la modernité de Behan à travers une comparaison entre Borstal Boy et Molloy de Beckett. Alors que Derek Hand et Maria DiBattista questionnent les aspects de l'identité et de l'autobiographie, Michael G. Cronin étudie, quant à lui, les thématiques de l'éros et de l'homosexualité et démontre comment l'espace prison devient, paradoxalement, un espace de liberté.

Concernant les six autres essais, deux explorent la question du bilinguisme. À travers un corpus de treize poèmes en gaélique, publiés entre 1946 et 1952 dans la revue Comhar, Rióna Ní Fhrighil s'interroge sur le choix de Behan d'utiliser le gaélique, choix qui s'avère moins lié à des raisons politiques qu'à la recherche artistique (p. 94) et qui est essentiel dans l'exploration de nouvelles formes d'expression (p. 115).

Clíona Ní Ríordáin se pose la question de la traduction, ou adaptation, de An Giall, et cherche à sortir de l'impasse où mènent les notions de pureté, infidélité et hybridité (p. 125) en assumant l'hypothèse d'une coécriture Behan-Littlewood pour la production de la version anglaise, The Hostage. Dépasser l'idée d'une hiérarchie entre les deux textes est aussi la préoccupation de Mícheál Mac Craith qui préfère élargir le spectre de lecture de la pièce en dehors du contexte irlandais à travers une étude comparative avec L'aube d'Elie Wiesel. 
Dans les deux essais qui suivent, signés respectivement par John McCourt et Paul Fagan, il est question des rapports entre Behan et deux figures majeures de la littérature irlandaise: James Joyce et Brian O’Nolan. Le premier s'appuie sur le texte d'une conférence donnée par Behan à New York en 1962 pour «explorer l'importante influence de Joyce sur Behan tant dans son rôle de chroniqueur de Dublin que d'auteur moderniste» (p. 12). Fagan, quant à lui, analyse la manière dont O’Nolan présente Behan, le personnage, dans les chroniques écrites sous le pseudonyme de Myles na Gopaleen dans les années 1950.

Pour terminer, Michael Pierse s'attaque à la dernière pièce de Behan, Richard's Cork Leg. Pierse est courageux car, comme pour d'autres textes de Behan, l'approche critique est problématique, la pièce ayant été reconstituée par Alan Simpson à partir des notes écrites par Behan entre décembre 1960 et juin 1961. Cette dernière contribution est peut-être la plus intéressante dans ce qu'elle soulève comme interrogation. Comment recevoir les derniers textes de Behan, incomplets ou altérés par l'intervention d'un tiers, mais qui peuvent apporter des clés nouvelles pour une plus ample compréhension de l'auteur?

McCourt le dit en introduction, beaucoup reste à dire sur Behan (p. 2) et cet ouvrage, à lui seul, ne pourrait combler le vide. Il est néanmoins inspirant et on y trouve aussi une bibliographie complète. La promesse de l'éditeur d'apporter une lumière nouvelle sur le travail de Behan est tenue, et nous partageons avec McCourt l'espoir qu'il puisse montrer le chemin et susciter un regain d'intérêt de la part de la critique (p. 14).

Chiara Rustici

Faith Binckes, Kathryn Laing, Hannah Lynch (1859-1904): Irish Writer, Cosmopolitan, New Woman, Cork, Cork University Press, 2019, xii + 248 p.

The recent decade in Irish literature has been marked by a boom in critical research that seeks to restore fairness to the canon's gender inequality. Although there is always a risk of exclusion, in the case of Ireland, women writers have suffered from a serious degree of under-representation in Irish critical discourse. The change is detectable by a new wave of inspiring critics who challenge previous traditions, showing the cultural richness of female presences in Irish literary history.

The late $19^{\text {th }} /$ early $20^{\text {th }}$ century was a crucial period in Ireland, marking the beginning of the free Irish State and the nation's cultural revival. However, as contemporary scholarly research shows, it was also a prosperous period of Irish-born women writers who actively participated in the re-formation of the nation. The long line of recent critical studies reflecting on this female literary achievement includes 